\title{
Presentación del número
}

\section{Presentación del número 2 volumen 5 de RIECS}

\author{
Manuel Rodríguez Zapata \\ Profesor Emérito del Departamento de Medicina y Especialidades Médicas, Universidad de Alcalá; \\ manuel.rodriguezz@uah.es; https://orcid.org/0000-0003-3673-4025
}

DOI: https://doi.org/10.37536/RIECS.2020.5.2.240

RIECS mantiene su pulso, a pesar de las circunstancias especiales en las que vive el mundo entero, nuestra sociedad, el sistema sanitario y la comunidad universitaria en particular.

Una de las formas de luchar, para sobrevivir a esta situación, es la de persistir, fuerte y constante, en la línea de trabajo que a todos nos corresponde, en la esperanza de superar en algún momento las dificultades y los cambios que nos están condicionando a todos, como individuos y como colectividad.

Este es el espíritu que ha impulsado la elaboración de este número de la Revista de Investigación y Educación en Ciencias de la Salud.

Este momento coincide, además, con el cambio necesario de la responsable al frente del Decanato de la Facultad y del Equipo Decanal. Por este motivo era imprescindible que la Decana actual de la Facultad de Medicina y Ciencias de la Salud de la Universidad de Alcalá, la Profesora Lourdes LLedó García, editorializara sobre el impacto que ha supuesto esta pandemia sobre la docencia universitaria, sobre la capacidad de adaptación de todos los colectivos que forman nuestra comunidad para mantener nuestra misión docente, discente, investigadora y de gestión de los recursos. Esta crisis, como todas, siguiendo un discurso "darwiniano", nos debe dar la oportunidad de dar un paso adelante en nuestros objetivos y permitirá descubrir territorios y métodos nuevos o poco explorados para mejorar nuestro trabajo.

Las aportaciones presentadas en este número reflejan el universo variado que constituye esta facultad y del que nosotros, como revista, somos un fiel testigo.

Así, en el apartado reservado a originales, el profesor Francisco Javier Burgos-Revilla y su grupo de colaboradores, de los Servicios de Urología y Nefrología del Hospital Universitario Ramón y Cajal, encabezados por Marta Santiago-González y Victoria Gómez Dos Santos nos presentan "Trasplante renal en pacientes con infección por virus de la inmunodeficiencia humana (VIH)" y concluyen que es una alternativa terapéutica segura y efectiva en pacientes seleccionados con VIH.

Las profesoras $\mathbf{M}^{\mathbf{a}}$ Victorina Aguiar, $\mathbf{M}^{\mathbf{a}}$ del Carmen Berrocal y Teresa Hernández, de la Unidad de Nutrición y Bromatología del Departamento de Ciencias Biomédica de la Universidad de Alcala, su artículo original "Nutrición y VIH/SIDA: cumplimiento de los Objetivos de Desarrolla Sostenible", describen un tema de vital importancia en el manejo integral de estos pacientes, y como la detección y evaluación temprana de los déficit nutricionales, asociados al TAR, han contribuido a disminuir la morbimortalidad asociada al VIH, permitiendo la consecución de los ODS.

Esta sección se completa con el excelente trabajo “Efectos agudos de una sesión de entrenamiento vibratorio sobre la espasticidad en personas con Esclerosis Múltiple: resultados preliminares", presentado por el profesor Luis Andreu-Caravaca, Jacobo A. Rubio-Arias y colaboradores de la Facultad de Deporte de la Universidad Católica San Antonio y de la Cátedra Internacional de Medicina del Deporte de dicha Universidad, en colaboración con el Departamento de Ciencias de la Actividad Física y del Deporte de la Universidad de Valencia, del Centro de Investigación para el Deporte de Alto Rendimiento de la Universidad Católica de Murcia y del Departamento de Salud y Rendimiento de la Facultad de Ciencias de la Actividad Física y del Deporte 
de la Universidad Politécnica de Madrid. Trabajo que concluye con la necesidad de individualización del entrenamiento vibratorio en la población con Esclerosis Múltiple.

Ángeles Martín Octavio. Directora de Enfermería del Hospital Universitario de Guadalajara. SESCAM, nos aporta en su Artículo de Opinión, "La gestión sanitaria en la segunda fase de la pandemia del coronavirus: Más difícil, si cabe", sus reflexiones sobre la forma de afrontar esta segunda fase de la pandemia desde el punto de vista de los pacientes, del personal sanitario y sobre todo desde el papel de los gestores sanitarios, subrayando la necesidad de liderazgo profesional, potenciación de la atención primaria, el papel de la epidemiología y de la salud pública, de las tecnología de la información y de la necesidad de crear modelos centrados en la atención al paciente.

El apartado de Revisiones describe aspectos actuales de una gran relevancia en diferentes campos del desarrollo de la investigación en enfermedades muy prevalentes y de un gran interés médico y social.

Los profesores Cecilio Álamo, Cristina Zaragozá y Francisco López-Muñon, del Departamento de Ciencias Biomédicas de la Facultad de Medicina y Ciencias de la Salud de la Universidad de Alcala y la Facultad de Ciencias de la Salud de la Universidad Camilo José Cela y de la Unidad de Neuropsicofarmacología del Instituto de Investigación del Hospital 12 de Octubre de Madrid, presentan un excelente trabajo "Algo más que monoaminas en el tratamiento de la depresión: Mecanismos neurobiológicos emergentes de los antidepresivos del siglo XXI" en el que profundizan en los nuevos mecanismos fisiopatológicos implicados en la depresión y en la búsqueda de dianas para la utilización de diferentes antidepresivos.

El profesor José Alcamí Pertejo, Profesor de Investigación del Instituto de Salud Carlos III, Director Científico de la Unidad VIH del Hospital Clinic de Barcelona y Director de la Cátedra RIS de la Universidad de Alcalá, analiza en su artículo “Una vacuna preventiva frente al VIH. Situación actual y perspectivas", las distintas etapas y estrategias seguidas, sin éxito, para conseguir una vacuna eficaz frente al VIH y propone el nuevo paradigma y los nuevos abordajes utilizados en el momento actual, frente a un virus complejo con diferentes mecanismos de escape de cualquier otro virus.

Por último, Verónica del Prado Sánchez, MIR y la Profesora Consuelo Giménez Pardo, Secretaria Académica de la Facultad de Medicina y Ciencias de la Salud y revisan la "Situación sanitaria de la población refugiada en los campos de desplazados de Grecia" concluyendo que por factores como la crisis económica y un sistema sanitario desigual e ineficaz, Grecia no cuenta con la capacidad necesaria para la recepción de población emigrante, en general, ni para poblaciones especialmente vulnerables como son las mujeres y los menores no acompañados.

En este número contamos con una colaboración muy especial, a la que hemos reservado el espacio del Aula Magna. Se trata del trabajo del Profesor Francisco Zaragozá García, Catedrático Emérito de Farmacología de la Universidad de Alcalá, que nos describe “El Plan Profarma y las células CAR-T". Las células CAR-T constituyen una auténtica revolución en terapia cancerosa y este plan, diseñado por los Ministerios de Industria y Sanidad, tiene como objetivo incentivar a las compañías farmacéuticas y dar la oportunidad a los grupos de investigación para realizar su trabajo en materia de medicamentos y de forma competitiva.

En el apartado de Notas Clínicas Laura Mola Reyes del Servicio de Endocrinología y colaboradores del Servicio de Nefrología del Hospital Central de la Defensa Gómez Ulla, nos presenta un caso de "Hiponatremia grave en Macroadenoma Hipofisario, ¿la infección por COVID-19 la exacerba?" en el que discute el proceso diagnóstico de este paciente y alerta sobre las repercusiones, en supervivientes de la infección por COVID-19, sobre la presencia de hipocortisolismo central. 
Sobre Metodología Docente y Evaluación, les presentamos dos trabajos relacionados con el aprendizaje y el razonamiento clínico.

El primero, realizado por la Profesora Ana María de Santiago Nocito, y el Profesor Alberto García Lledó, Profesores Asociados del Departamento de Medicina y Especialidades Médicas de la Universidad de Alcalá sobre "EL SCRIPT CONCORDANCE TEST como herramienta evaluativa" en el que se describe el proceso de construcción de este tipo de preguntas y su sistema de evaluación. En el segundo artículo, "Nuevas fórmulas en la gestión del proceso educativo",

José Antonio Martínez-Pérez, Doctor en Medicina, nos revisa, a partir del centenario del Informe Flexner, los documentos emergidos en la última década, el informe Carnegie 2010 y el Consenso Global sobre responsabilidad social de la Facultad de Medicina, como referencia de futuro en la Educación Médica.

Los miembros de la Delegación de estudiantes de Ciencias de la Actividad Física y del Deporte de la Universidad de Alcalá, Sergio Fernández Lobo, Paloma Arenas y Jaime Ballesteros, nos describen, en una Carta al Director lo que supone y ha supuesto "Ser representante estudiantil en tiempos de pandemia".

Y por fin llegamos a Ágora, nuestro espacio de reflexión, ocio y creatividad, donde reencontrarnos con una realidad distinta y reconfortante y con una poesía de nuestra secretaria del decanato Dña. María Soledad Pastor Palomo.

Como siempre, espero que les guste $i i i$

(C) 2020 por los autores; Esta obra está sujeta a la licencia de Reconocimiento 4.0 Internacional de Creative Commons. Para ver una copia de esta licencia, visite http://creativecommons.org/licenses/by-nc-nd/4.0/. 